\title{
ON A THEOREM OF ERDÖS AND SZEMEREDI
}

\author{
By M. J. NARLIKAR
}

\section{\$. Introduction}

In 1951, K. F. Roth [ 7] proved that if $1=q_{1}<q_{2}<\ldots$. is the sequence of all square - free integers, then

$$
q_{n+1}-q_{n}=o\left(n^{\frac{3}{13}}(\log n)^{\frac{4}{13}}\right)
$$

and this was improved to $O\left(n^{\frac{2}{9}}\right)$ by H. E Richert [6]. In an attempt to put these in more general setting P. Erdö [1] introduced with any sequence $\mathrm{B}: 2<b_{1}<b_{2}<\ldots \ldots \ldots$, the sequence $Q: 1-q_{1}<q_{2}$. of all integers $q_{i}$ not divisible by any $b_{j}$ and proved (subject only to $\left(b_{i}, b_{j}\right)=1$ ualess $i=j$ and $\left.\sum \frac{1}{b_{i}}<\infty\right)$ that

$$
q_{n+1}-q_{n}=o\left(q_{n}^{0}\right) \text {, }
$$

with some $\theta<1$, where $\theta$ is independent of $B$. His $\theta$ was close to 1. E. Szemeredi made an important progress and showed that this is true for every fixed $\theta>\frac{1}{2}$. As in all previous results of this kind, he showed that if $Q(x)=\underset{q_{i}}{\Sigma}<x$, then $Q(x+h)-Q(x) \gg h$, where $h>{ }_{8} \theta$. 
Using the ideas of Szemeredi with some refinements, we prove

\section{Theorem I}

Let $p$ be any prime and $r_{p}$ denote the number of $b_{i}$ divisible by $p$ and suppose that as $p$ varies, $r_{p}$ does not erceed $p^{d}$ where $A$ is any positive constunt. Let $\Sigma \frac{1}{b_{i}}<\infty$. Then

$$
Q(x+h)-Q(x) \gg h, \text { where } x>h>x^{\theta}
$$

and $\theta>\frac{1}{2}$ is any constant.

Further if for some $\alpha<1$, we have $\Sigma b_{i}^{-\alpha}<\infty$, then

$$
Q(x+h)-Q(x) \gg h \text {. }
$$

where $h>x^{\theta}$, and $\theta>\frac{\alpha}{1+\alpha}=\beta$ say.

Next using the ideas of Jutila [4], the results of Brun ['] and the zero-free region for $\zeta(s)$ due to I M. Vinogradov [9] we prove

\section{Theorem 2}

$$
\begin{aligned}
& \text { Let } r_{p} \leqslant p^{A} \text { as before and in place of } \\
& \Sigma b_{i}^{-1}<\infty \text { let } \lim _{y \rightarrow \infty} \Sigma_{i} b_{i} \leqslant y^{2} b_{i}^{-1}=0 \text {. Then } \\
& \qquad(x+h)-Q(x) \gg h / \log x,
\end{aligned}
$$

The improvement Theorem 2 of Theorem 1 was suggested by Professor K. Ramachandra and I am thankful to him for explaining the same. Also I express my gratitude to him for encouragement and useful suggestions. He and $I$, in a joint paper to appear have improved Theorem 2 in several ways These researches will appear in Acta Arithmetica in due course. 


\section{2. Proofs of Theorems $1 \& 2$}

We begin with some notations.

(I) We can assume without loss of generality that $b_{1}, b_{2}, \ldots b_{k}$ are primes (because we can replace them by their greatest prime factor and select distinct ones amongst them). Next we assume

$$
\begin{aligned}
& \underset{i>k}{\Sigma} b_{i}-1<\frac{1}{2} \text { and define } j_{0} \text { by } \\
& i j_{0}^{\Sigma} b_{i}^{-1}<\eta \text {, where } \eta \text { is sufficiently small. }
\end{aligned}
$$

(2) Let $n>10$ be any large integer constant and for $i=1,2, \ldots n$ put

$$
\begin{aligned}
& C_{i}=\left\{p / x^{(2 n)-1}+(i-1)\left(8 n^{4}\right)^{-1}<p\right. \\
& \leqslant x^{(2 n)^{-1}}+i \overline{\left(8 n^{i}\right)}-1 \\
& C_{i}^{\prime}=\left\{p \mid x^{\beta(2 n)^{-1}}+(i-1)\left(8 n^{4}\right)^{-1}<p\right. \\
& \left.\leqslant x^{\beta(2 n)^{-1}}+i\left(8 n^{4}\right)^{-1}\right\} \text {. }
\end{aligned}
$$

Let $g$ run over integers of the form $\pi p$, where $p$ are chosen one from each $C_{i}$. Let $g_{i}$ be defined in the same way with respect to $C_{i}{ }^{\prime}$. For any fixed integer $g$ the number of integers in $[x, x+h]$ which are divisible by $g$ (respectively $\left.g b_{i}\right), k<i \leqslant j_{0}$ but coprime to $b_{1}, b_{2}, \ldots b_{k}$ is

$$
\begin{gathered}
\frac{h}{g} \underset{i \leqslant k}{\pi}\left(1-\frac{1}{b_{i}}\right)+O\left(2^{k}\right) . \\
\text { (respectively } \left.\frac{h}{g b_{j}} \underset{i \leqslant k}{\pi}\left(1-\frac{1}{b_{i}}\right)+O\left(2^{k}\right)\right)
\end{gathered}
$$


Also the number of integers in $[x, x+h]$ divisible by $b_{i}$ for any fixed $i \geqslant j_{0}$ such that $b_{i}<h$ is $<\frac{2 h}{b_{i}}$. Hence the number of integers (counted with certain multiplicities) in $[x, x+h]$ which are coprime to $b_{1}, b_{2}, \ldots b_{k}$, but divisible by some $g$ or other, but not divisible by any $b_{i}\left(i>j_{0}, b_{i} \leqslant h\right)$

$$
\begin{aligned}
& >\sum_{g}\left\{\frac{h}{g} \sum_{i \leq k}\left(1-\frac{1}{b_{i}}\right)-\right. \\
& \left.\sum_{k<i<j_{0}}\left(\frac{h}{g b_{i}} \underset{i<k}{\pi}\left(1-\frac{1}{b_{i}}\right)+o\left(2^{k}\right)\right)\right\} \\
& -\sum_{b_{0}{ }_{0}^{-r}<b_{j}<h} \frac{2 h}{b_{i}}
\end{aligned}
$$

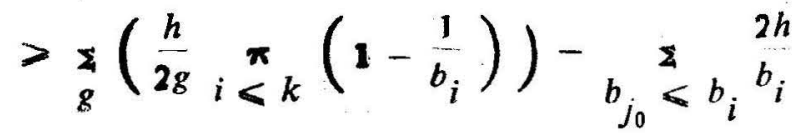

$$
\begin{aligned}
& +o\left(2^{k} j_{0} \sum_{g}^{\Sigma 1}\right) \\
& =h\left\{\frac{1}{2}\left(\pi\left({ }_{i}^{\sum} \sum_{i} \frac{1}{p}\right)\right) \underset{i<k}{\pi}\left(1-\frac{1}{b_{i}}\right)-2 \eta\right\} \\
& +O\left(2^{k} j_{0} x^{\frac{1}{2}}+\frac{1}{8 n^{2}}\right) \\
& >h\left\{\left(\frac{1}{1000 n}\right)^{10 n} i_{i<k}^{\pi}\left(1-\frac{1}{b}\right)-2 \eta\right\} \\
& +O\left(2^{k} j_{0} x^{\frac{1}{2}+\frac{1}{8 n^{2}}}\right)
\end{aligned}
$$


The multiplicities are $<(4 n) !$ since the number of prime factors $>x^{\frac{1}{2 n}}$ is $\leqslant 4 n$ for the integers counted. Now let us look at integers in $[x, x+h]$ which are of the form $m g$ but divisible by some $b_{i}$ or the other with $b_{i}>h$. Now a given $b_{i}$ can divide atmost one integer in the interval and so it suffices to count the number of $b_{i}$ to get an upper bound for the number of integers in question If $\left(b_{i}, g\right)=1$, then $b_{i} g<2 x$ and hence $h g: \leqslant 2 x$. This is impossible since $h>x^{\theta}$. Hence $\left(b_{i}, g\right)>1$ and the number of possible $b_{i}{ }^{\prime} s$ is therefore

$$
<\sum_{p<x^{\frac{1}{n}}} p^{A}<x^{\frac{A+1}{n}}
$$

A large choice of $n$ now completes the proof of the first part of Theorem 1. The second part can be proved similarly using $C_{i}{ }^{\prime}$.

The proof of Theorem 2 to put it briefly starts with

$$
\begin{array}{r}
\frac{1}{2 \pi i} \int\left(-\sum_{p}\left(p^{-s} \log p\right)\right)\left(\sum_{X<p<2 X} p^{-s}\right)^{N} \\
\times \frac{(x+h)^{s}-x^{s}}{s} d s
\end{array}
$$

where the line of integration is $\sigma=1+(\log x)^{-1}$.

$|t|<T$. We then move the line of integration to $\sigma=1-(\log T)^{-\frac{99}{100}}$. Rough estimations are enough to 
show that the number of numbers of the form $p p_{1} p_{2} \ldots \ldots p_{N}$ $\left(X \leqslant p_{i} \leqslant 2 X\right)$ lying in $[x, x+h]$ is $\gg \frac{h}{\log x}$ (provided $X=T^{\frac{1}{n}}$ and $N=n-1$ and $h=x^{\frac{1}{2}+\eta}$ where $\eta>0$ is small provided $n$ is large). If $b_{i}$ divides a number of the counted type then $x^{\frac{1}{2 n}} \leqslant b_{i}<x^{2}$ and by Brun's sieve the nu nber of cousted au nobers divisible by such $b_{i}$ is

$$
\begin{aligned}
\sum_{x^{2 n}}^{\frac{1}{2 n}} & \sum_{i}<\frac{h}{(\log x)^{2}}\left(\frac{h}{b_{i} \log x}+1\right)+ \\
& \sum^{\prime} b_{i} \leqslant x^{2}
\end{aligned}
$$

where the second sum is over those $b_{i}$ which divide a number of the counted type. This proves Theorem 2.

Remark: In the joint paper [5] to appear, the present paper has been referred to under the title "An Analytic Approach to Szemeredi's Theorem ". The interested readers will please note this change of title. The paper was written in 1977 and could not be published earlier because of some reasons. 


\section{References}

1. P. Erdös, On the difference of consecutive terms of sequences defined by divisibility properties, Acta Arith. 19 (1966). $175-182$.

2. H. Halberstam and H. E. Richert, Sieve methods, Academic Press (1974)

3. H. Halberstam and K. F. Roth, On the gaps between consecutive square - free integers, J. London Math. Soc., $26(1951), 268-273$.

4. M. Jutila, On numbers with a large prime factor, $J$. Indian Math. Soc., 37 ( 1973), 43 - J3.

5. M. J. Narlikar and K. Ramachandra, Contributions to the Erdos - Szemeredi theory of sieved integers, Acta Arith., ( to appear)

6. H. E. Richert, on the difference between consecutive square-free numbers, J. London Math Soc., 29 (1954), 16-20.

7. K F. Roth, On the gaps between square-free numbers, J. Londun Math. Soc, 26(1951), $263-268$.

8. E. Szemeredi, on the difference of consecutive terms of sequences defined by divisibility properties-II, Acta Arith., 23 (1973), 359 - 361.

9. E C. Titchmarsh. The theory of the Riemann zeta function, Clarendon Press, Oxford (1951).

701. Colabs Housing Colony,

Homi Bhabha Road,

Bombay 400005

India. 